\title{
Eine kleine Abhandlung über Methoden und ihre Formen in der EU-Praxis
}

\author{
Antonio Missiroli*
}

Die jüngsten Richtungsdebatten über die Zukunft des europäischen Integrationsprozesses - nach Inkrafttreten des Vertrags von Lissabon und dem Beginn der Krise der Eurozone wurden verhältnismäßig polarisiert geführt: zwischen den Verteidigern und Hütern der Gemeinschaftsmethode (GM) auf der einen Seite und auf der anderen Seite den Fürsprechern eines stärker intergouvernemental ausgerichteten Ansatzes - die darin entweder einen wünschenswerten Trend oder eine unvermeidliche Kurskorrektur sehen.

Insbesondere in der Rede, die die deutsche Bundeskanzlerin Angela Merkel am 2. November 2010 am College of Europe in Brügge hielt - wo bereits in der Vergangenheit ähnlich wegweisende Einlassungen gemacht wurden, ${ }^{1}$ wurde der Begriff der „Unionsmethode“ (UM) eingeführt. Die UM gilt demnach als stärker im Einklang mit den aktuellen Begebenheiten und den politischen Entwicklungen in der Europäischen Union stehend, insbesondere was die Rolle der Mitgliedstaaten betrifft. Merkel definierte die UM allerdings lediglich als „ein koordiniertes Vorgehen im Geiste der Solidarität“ - und führte die Energiepolitik zur Illustration an. ${ }^{2}$

Nomina sunt consequentia rerum, Namen sind die Folge von Dingen, so ist man versucht mit Thomas von Aquin zu sagen. In den internationalen Medien wurde nun reichlich darüber spekuliert, ob Deutschland - seit Jahrzehnten der wichtigste Verteidiger einer ,immer engeren“ Union, der besonderen Rolle der Gemeinschaftsorgane/EU-Organe und einer regelbasierten Ordnung - der Brüsseler Exekutive den Rücken gekehrt hat und all sein Geld (nicht nur metaphorisch) dem Europäischen Parlament, vor allem aber dem Rat als auch (im Prinzip) der Europäischen Zentralbank zukommen lässt. ${ }^{3}$

Man ist auch versucht zu sagen, dass diese Polarisierungen und Charakterisierungen vor allem als Instrument genutzt und immer dann vorgebracht werden, wenn es darum geht, wegweisende Entscheidungen auf europäischer Ebene in die eine oder andere Richtung zu lenken.

* Dr. Antonio Missiroli, Berater in der Outreach Section des Bureau of European Policy Advisers der Europäischen Kommission, Brüssel.

Dieser Beitrag gibt ausschließlich die persönliche Auffassung des Verfassers wieder.

Eine frühere englische Fassung dieses Beitrags ist unter dem Titel „A little discourse on method(s)“ als European Policy Brief 2 im Juni 2011 bei EGMONT - The Royal Institute for International Relations erschienen.

1 Beginnend mit Margaret Thatcher im Jahr 1988. Vgl. British Embassy - Press Service: Speech to be Given by the RtHon Mrs Thatcher Frs, Mp, At the Opening of the 39th Academic Year of The College of Europe, Bruges: 20 September 1988, abrufbar unter: http://www.coleurope.eu/file/content/news/Speeches/1988-1989.pdf (letzter Zugriff: 26.09.2011).

2 Rede von Bundeskanzlerin Merkel anlässlich der Eröffnung des 61. Akademischen Jahres des Europakollegs Brügge. Di, 02.11 .2010 in Brügge, abrufbar unter: http://www.coleurope.eu/file/content/news/Speeches/ Rede\%20Merkel\%20Europakolleg\%20Bruegge.pdf (letzter Zugriff: 26.09.2011).

3 Es ist nicht nur eine Frage der Gewichtung, sei es, dass diese nun bemessen wird anhand der Sitze im Europäischen Parlament, der (neu-)gewichteten Stimmen im Rat oder anhand des Einflusses der Bundesbank: Ein Deutscher ist Generalsekretär des Europäischen Parlaments (Klaus Welle), ein anderer ist Generalsekretär des Rates (Uwe Corsepius, ein enger Vertrauter von Kanzlerin Merkel). 
Nun ist es in der Tat so, dass keine der Methoden den gegebenen Machtverhältnissen und den Entscheidungsprozessen der heutigen Europäischen Union gerecht wird - diese sind weitaus komplexer und es ist bedauerlicherweise zweifelsohne schwierig, diese in einer einzigen, allumfassenden Formel auf den Punkt zu bringen. Insofern ist die US-amerikanische Devise, e pluribus unum, hier kaum anwendbar.

Selbst in der Vergangenheit, insbesondere während der beiden letzten Jahrzehnte, entsprachen die sogenannten supranationalen und intergouvernementalen Ansätze eher Idealtypen im Sinne von Max Weber und nicht eindeutigen Methoden oder Modellen - und sie tauchten nur selten in ,reiner', unverfälschter Form auf. Dennoch hatten sie lange eine Monopolstellung und eine polarisierende Wirkung, sowohl in der akademischen Forschung wie auch im öffentlichen Diskurs.

Vielleicht kann es gerade deshalb sinnvoll sein, sich anderen Methoden und Philosophen zuzuwenden - beginnend mit René Descartes, mit seinem Drang den acquis von Aquin zu , dekonstruieren', kombiniert noch mit einigen empirischen Belegen à la David Hume ${ }^{4}$, um den Nebel zu durchdringen und die Mythen zu enthüllen, die sich derzeit in der öffentlichen Debatte in und über die Europäische Union ranken.

\section{Ex uno plures? Von der Gemeinschaftsmethode zur Methodenvielfalt}

What's in a name?, ließ William Shakespeare bekanntermaßen einst seine Julia zu ihrem Romeo sagen. In der Tat ist eine eindeutige Definition und allgemein anerkannte Beschreibung der GM schwer zu finden.

Vor ein paar Jahren beschrieb Helen Wallace die, wie sie sie nannte, ,traditionelle“ GM basierend auf dem Präzedenzfall, wie er anfangs mit der Gemeinsamen Agrarpolitik (GAP) geschaffen wurde - wie folgt:

- der Kommission wird eine starke Rolle zugewiesen bei der Gestaltung, Vermittlung und Ausführung der Politik und ihrer Außenwirkungen;

- eine Stärkung der Rolle des Rates durch strategisches Verhandeln und das Schnüren von Verhandlungspaketen;

- ein Einbinden von sektoralen Interessengruppen durch ein äußerst gewinnbringendes Belohnungssystem in den EU-Prozess;

- die Einbeziehung von nationalen Agenturen als untergeordnete und ausführende Organe des vereinbarten gemeinsamen Regimes;

- eine Begrenzung des Einflusses nationaler Abgeordneter und des Europäischen Parlaments;

- ein gelegentliches (doch klar bestimmendes) Eingreifen des Europäischen Gerichtshofs zur Stärkung der rechtlichen Befugnisse des Gemeinschaftssystems;

- kollektive Bereitstellung von Finanzmitteln als Ausdruck der fortwährenden europäischen Solidarität.

Dieses Muster wurde zum Inbegriff des ,supranationalen` Regierens, bei dem Machtbefugnisse von der nationalen Ebene auf die europäische Ebene übertragen wurden. Wie weit dieses tatsächlich mit der Realität übereinstimmt, ist ein strittiger Punkt, selbst bei der GAP und bereits die Fischereipolitik, die die GAP nachahmen sollte, entwickelte sich anders.

4 Der Discours de la méthode (1637) von René Descartes gilt allgemein als Wendepunkt in der Geschichte moderner Philosophie und steht für den Beginn des Rationalismus. A Treatise of Human Nature (1739) von David Hume bildet dazu mit einem starken Plädoyer für den Empirismus einen Gegenpol. 
Dennoch ist es diese bestimmte Definition der GM, die die, Vorstellung ' sowohl von Praktikern und Kommentatoren für eine ganze Weile prägte. ${ }^{5}$

Wallace betonte außerdem die Tatsache, dass die Europäischen Gemeinschaften/Europäische Union seit Mitte der 1980er Jahre begonnen hat, mindestens zwei zusätzliche Methoden anzuwenden.

Zunächst sei der Ruf nach und die Hinwendung zu mehr Regulierung genannt. Beides wurde mit der Etablierung des Wettbewerbsregimes und des Binnenmarkts immer wichtiger. Die Stärke des europäischen Gesetzgebungsprozesses, die Maschinerie zur Förderung der sachbezogenen Zusammenarbeit und die verhältnismäßig große Distanz zu parlamentarischer Mitwirkung waren alle Faktoren, die diesen Trend weiter förderten. Tatsächlich waren die Voraussetzungen der Europäischen Gemeinschaften/Europäischen Union besonders geeignet, einen übergreifenden Rechtsrahmen zu schaffen, der in der Lage war, grenzüberschreitende Standards mit länderspezifischen Eigenheiten zu kombinieren.

Die Hauptkennzeichen dieser regulativen Methode umfassten:

- die Kommission als Architektin und Verteidigerin (in quasi-richterlicher Funktion) der ordnungspolitischen Ziele und Regeln, oft in Verbindung mit Interessengruppen und Experten;

- der Rat als (Diskussions-)Forum für die Vereinbarung von Mindeststandards und die richtungsgebenden Entscheidungen für die Harmonisierung, ergänzt durch die gegenseitige Anerkennung von Präferenzen und Kontrollen;

- der Europäische Gerichtshof als Hauptinstanz zur Gewährleistung einer verhältnismäßig fairen und gerechten Rechtsdurchsetzung;

- das Europäische Parlament als eine von mehreren Institutionen zur Berücksichtigung auch nicht-ökonomischer Faktoren, mit wachsenden Gesetzgebungskompetenzen - bei gleichzeitig nur geringem Einfluss auf die tatsächliche Implementierung.

Hinzu kommt, dass sich im Laufe der Jahre der EU-Entscheidungsprozess zunehmend in Verhandlungen und Kontroversen über Verteilungsfragen als Folge der Integration verfangen hat. In diesem andauernden Prozess - der indirekt auch die GAP betraf - markiert die Einführung der Kohäsionspolitik eine Verlagerung hin zu Programmen zur Bekämpfung der wirtschaftlichen und sozialen Unterschiede sowie zur Unterstützung der rückständigen Regionen und/oder gesellschaftlichen Gruppen.

Darüber hinaus wurden auch in anderen Bereichen etliche ausgabewirksame Instrumente ins Leben gerufen, unter anderem im Bereich Forschung, die eher programmatische statt redistributionale Ziele verfolgen.

Zusammengenommen umfasste diese distributionale Methode:

- die Kommission als ,Erfinderin“ von Programmen, in Partnerschaft mit innerstaatlichen Behörden und/oder sektoralen Interessengruppen und Agenturen;

- die nationalen Regierungen, die sich im Rat (unter dem Druck der verschiedenen Behörden und Interessengruppen) auf einen Haushaltsrahmen mit einigen distributiven Elementen einigen;

- ein Europäisches Parlament, in dem die Abgeordneten mit Rücksicht auf das Wohlergehen ihrer Wahlkreise oft zusätzlichen Druck ausüben;

5 Helen Wallace: An Institutional Anatomy and Five Policy Modes, in: Helen Wallace/William Wallace/Mark A. Pollack (Hrsg.): Policy-Making in the European Union, 5. Auflage, Oxford/New York 2005, S. 49-90. Siehe aktuell Renaud Dehousse (Hrsg.): The 'Community Method': Obstinate or Obsolete?, Basingstoke/New York 2011. 
- lokale und regionale Behörden, die von einer Teilhabe am EU-Prozess profitieren und sich (seit 1993) auf ihre eigene Institution berufen können, den Ausschuss der Regionen.

Es war übrigens diese Öffnung hin zu einer unmittelbaren Interaktion zwischen der europäischen und der subnationalen Regierungsebene, die zur allgemeinen Prägung des Begriffs der Mehrebenenpolitik (,multi-level governance') zur Charakterisierung des EU-Prozesses führte.

\section{Weiter im Methodenspektrum wie gehabt?}

Schließlich lässt sich darüber diskutieren, ob - insbesondere im letzten Jahrzehnt - das Methodenspektrum durch die sogenannte Offene Methode der Koordinierung (OMK) erweitert wurde, die in der Regel mit der Lissabon-Strategie aus dem Jahr 2000 in Verbindung gebracht wird. Diese umfasst, weiche` Formen politischer Anreizsysteme, um das Handeln auf nationaler Ebene durch das sogenannte Benchmarking und den methodischen Politikvergleich mitzugestalten, allerdings ohne konkrete Durchsetzungs- und Implementierungsinstrumente zur Verfügung zu stellen.

Die EU-Beschäftigungspolitik ist ein weiteres typisches Beispiel hierfür - wenn auch mit einigen Abweichungen.

Die OMK kann als beinahe deckungsgleich mit der Intergouvernementalen Methode (IM) gelten - oder zumindest als eine Variation von dieser. Allerdings gilt sie zumindest aus politikfeldbezogener Perspektive in erster Linie für Bereiche des ehemaligen ersten ,Pfeilers'. Rückblickend hat sich ihr Erfolg allerdings als recht bescheiden erwiesen, wie die meist kritische Bilanz der Lissabon-Strategie bis zum Jahr 2010 zeigt.

Die, wie man sie nennen könnte, ,traditionelle' IM ihrerseits schließt ein:

- die aktive Mitwirkung des Europäischen Rates bei der Verabschiedung richtungsweisender Beschlüsse;

- die Dominanz des Ministerrates (oder eines Pendants) bei der Konsolidierung der Zusammenarbeit;

- die begrenzte/marginale Rolle der Kommission, im Vergleich zur GM und den anderen oben genannten Methoden;

- die grundsätzliche Nichtbeteiligung des Europäischen Parlaments (abgesehen von der Verabschiedung des Haushalts) und des Europäischen Gerichtshofs in den Phasen des Entscheidungsprozesses von der Politikformulierung über die Durchführung bis zur Kontrolle;

- die Einführung von eigenen Regeln für die Gestaltung der Zusammenarbeit (insbesondere im Generalsekretariat des Rates);

- die verhältnismäßig große Intransparenz des Entscheidungsprozesses, insbesondere für die nationalen Parlamente und die Bürger;

- die Fähigkeit, in bestimmten Bereichen substanzielle gemeinsame Politikentscheidungen zu treffen, die bis dahin von gemeinsamen Initiativen ausgeschlossen waren.

Auch hier wird deutlich, dass sich die IM in einer Reihe von signifikanten Variationen präsentiert - vor allem dann, wenn man so unterschiedliche Bereiche wie die Gemeinsame Außen- und Sicherheitspolitik (GASP) beziehungsweise die Gemeinsame Sicherheits- und Verteidigungspolitik (GSVP) sowie Justiz und Inneres berücksichtigt, die nach dem Vertrag von Maastricht den zweiten beziehungsweise dritten ,Pfeiler' der EU-Konstruktion bildeten.

In beiden Fällen standen (und stehen) die Ressourcen, Fähigkeiten und sogar Besitzstände der Mitgliedstaaten im Vordergrund. Dennoch ist es, selbst im erstgenannten Fall der europäischen Außenpolitik schwierig, diese als eindeutig intergouvernemental zu klassifi- 
zieren: Die Rolle, die Javier Solana in seinem Jahrzehnt als Hoher Vertreter der Europäischen Union für die Gemeinsame Außen- und Sicherheitspolitik (1999-2009) einnahm, ging oft weit über die Verkörperung des kleinsten gemeinsamen Nenners der Mitgliedstaaten hinaus. Auch die daraus entstandenen Strukturen sind heute ,hybrider ${ }^{6}$ als sie es zuvor waren. ${ }^{7}$ Darüber hinaus kam der Kommission eine entscheidende Rolle zu. Diese führte spezifische Politiken in den Bereichen Außenbeziehungen, Entwicklung und humanitäre Hilfe sowie insbesondere in der Handelspolitik durch - und erst recht im Handlungsfeld der Erweiterungspolitik, die als eine Art von Außen- und Sicherheitspolitik ,mit anderen Mitteln gelten kann (diesmal frei nach Carl von Clausewitz).

Mit der Zeit haben sich auch im Bereich Justiz und Inneres verschiedene Methoden herausgebildet, die heute noch teilweise nebeneinander bestehen. Das Schengen-Abkommen wurde zunächst bewusst außerhalb der EU-Verträge unterzeichnet und umgesetzt, bevor es schließlich zu einem späteren Zeitpunkt in das Vertragswerk integriert wurde. Darüber hinaus förderten wachsende Migrationsströme sowie die gestiegenen Herausforderungen durch den internationalen Terrorismus und grenzüberschreitende Kriminalität die Nachfrage nach einer politischen Zusammenarbeit.

Dies hat dazu geführt, dass verschiedene Kooperationsverfahren zusammengeführt wurden, einschließlich der Überführung einiger Themen des Bereichs Justiz und Inneres in den (jetzt alten) ersten ,Pfeiler'. Insgesamt bleibt weiterhin ein gemischtes Bild von dem bestehen, was von Wallace als „Trans-Governmentalismus“ beschrieben wurde. Es wird ergänzt durch eine wachsende Zahl von spezialisierten Agenturen und rechtlichen Vereinbarungen, die auf Übereinkommen basieren - von der sogenannten Komitologie ganz zu schweigen.

Auch die Europäische Währungsunion ist ein weiteres interessantes und erwähnenswertes Beispiel. Auf der einen Seite haben die Europäische Zentralbank und die anderen einschlägigen Organisationen für die Währungspolitik eine Variante der GM geschaffen (in der die Europäische Zentralbank als funktionelles quasi-Äquivalent der Kommission fungiert). Auf der anderen Seite - nämlich soweit es die Wirtschaftspolitik betrifft - besteht seit geraumer Zeit etwas Vergleichbares zur OMK. Die Rolle der Kommission hat sich im gesamten wirtschaftspolitischen Bereich über die Jahre hinweg gewandelt, abhängig vom Willen und der Bereitschaft der Mitgliedstaaten (allen voran Deutschland), diese mit einzubeziehen zumindest bisher.

Ebenfalls erwähnenswert ist, dass es sich bei den drei genannten Bereichen - GASP, Justiz und Inneres sowie Europäische Währungsunion - um die mit Abstand dynamischsten Bereiche der Europapolitik seit 1999 handelt. Grundsätzlich wurde in allen drei Bereichen die Zusammenarbeit im EU-Rahmen nach und nach akzeptiert, während allerdings die jeweiligen institutionellen Arrangements gleichzeitig immer untypischer wurden.

Ferner muss auch gesagt werden, dass all diese Methoden dazu neigen, sich zu überlappen und - so lässt sich das wohl formulieren - sich hin und her zu bewegen, als eine Reaktion auf neue politische Herausforderungen sowie sich wandelnde Präferenzen und Feedback-Effekte der Mitgliedstaaten.

Solche Überlappungen und ,Wanderungen' treten nicht nur über die Pfeilergrenzen hinweg in Erscheinung, sondern auch innerhalb der alten ,Pfeiler'. So entsteht ein Flickenteppich von Modalitäten und Verfahren, der von außen (vor allem für den normalen EU-Bür-

6 Gisela Müller-Brandeck-Bocquet/Carolin Rüger: Zehn Jahre Hoher Vertreter - Lehren für die EU-Außen- und Sicherheitspolitik nach Lissabon, in: integration 1/2011, S. 24-41.

7 Siehe die jüngste Neubewertung von Jolyon Howorth: Decision-Making in Security and Defence Policy: Towards Supranational Intergovernmentalism?, Kolleg-Forschergruppe "The Transformative Power of Europe": KFG Working Paper, Nr. 25, März 2011. 
ger) oft nur mühsam beziehungsweise nicht zugänglich ist. Darüber hinaus erzeugen diese Wandlungsprozesse nicht immer ein und dieselbe Wirkung: Während eine Reihe von Politikbereichen tatsächlich zunehmend, vergemeinschaftet " wurde (wenn auch in unterschiedlichem Grad), hat sich bemerkenswerter Weise insbesondere die Energiepolitik in die entgegen gesetzte Richtung bewegt - vor allem im Vergleich zu den 1950er und 1960er Jahren.

Ein weiterer Punkt, den es in diesem Zusammenhang zu berücksichtigen gilt, ist die zunehmende Betonung - auch innerhalb des Vertragswerks - der sogenannten Subsidiarität. Im Nachhinein kann dies als deutliche Warnung der Mitgliedstaaten - nicht nur der Regierungen, sondern auch der nationalen Parlamente und sogar einiger regionaler Akteure - an die Kommission verstanden werden, ihren Kompetenzbereich nicht auf Bereiche auszuweiten, die primär in nationale Zuständigkeiten fallen. Einer Ausdehnung der GM Einhalt zu gebieten, bedeutet auch einige Politikbereiche von der Rechtsprechung des Europäischen Gerichtshofes auszuklammern.

Schlussendlich, aber keineswegs weniger wichtig, wurde mit der Einführung der abgestuften Integration in Gestalt der verstärkten Zusammenarbeit - vom Vertrag von Amsterdam (1999) bis zum Vertrag von Lissabon (2009) - eine weitere Methode in den Vordergrund gerückt: nämlich eine, die politikfeldübergreifend ist (wenn auch jeweils mit erheblichen Unterschieden) und an der Grenze zwischen einem Entscheidungs- und Implementierungsverfahren liegt. In seinem früheren (akademischen) Leben machte der finnische Minister für europäische Angelegenheiten und Außenhandel Alexander Stubb eine entscheidende und fast prophetische Unterscheidung zwischen „vordefinierten“ und ,ermächtigenden“"Klauseln der verstärkten Zusammenarbeit. ${ }^{8}$

Die Europäische Währungsunion ist so ein Fall - in gewisser Weise ante litteram - da bereits vorab ihr Wirkungsbereich, die Teilnahmebedingungen und die Verfahren der Zusammenarbeit definiert wurden. Was der Vertrag über die Europäische Union (EUV) als ,ständige strukturierte Zusammenarbeit ${ }^{“ 9}$ im Verteidigungsbereich benennt, ist ein potenziell weiterer Fall - wenn auch in einer weniger bindenden Ausführung, mit weniger präzisem Umfang und ohne klar ersichtliche Verpflichtung oder einer Frist für die Umsetzung.

Die „ermächtigende“ Form der verstärkten Zusammenarbeit dürfte am ehesten im Bereich Justiz und Inneres Anwendung finden: Sie wurde zum ersten Mal Ende 2001 mit dem Europäischen Haftbefehl angedroht (der allerdings nicht umgesetzt wurde und stattdessen zu einem Kompromiss der 15 Mitgliedstaaten führte); kürzlich wurde sie bei der einheitlichen Regelung für grenzüberschreitende Scheidungen durchgesetzt.

Grundsätzlich ist jedoch die ,ermächtigende“ Form der verstärkten Zusammenarbeit auch in anderen Bereichen anwendbar - wie es heute der Fall ist bei der EU-weiten Regelung des Patentwesens und wie es eines Tages mit der gemeinsamen Bemessungsgrundlage einer Körperschaftsteuer möglich sein könnte. Und es ist nicht ganz auszuschließen, dass die differenzierte Integration (oder eine flexible beziehungsweise eine Integration der verschiedenen Geschwindigkeiten) - innerhalb oder außerhalb der verstärkten Zusammenarbeit eine immer wieder angewendete Methode für eine immer größere Union wird. Die jüngsten Entwicklungen in der öffentlichen Debatte über die Stärkung der Governance der Eurozone weisen eben in diese Richtung.

8 Alexander C.-G. Stubb: A Categorisation of Differentiated Integration, in: Journal of Common Market Studies 2/1996, S. 283-295.

9 Vgl. Art. 42 und 46 EUV sowie Protokoll (Nr. 10) über die ständige strukturierte Zusammenarbeit nach Artikel 42 des Vertrags über die Europäische Union, in: Amtsblatt der EU, Nr. C 83 vom 30. März 2011, S. 275-277. 


\section{Lissabon und die Zeit danach: Einheit in Vielfalt?}

Mit dem Vertrag von Lissabon hat sich in gewisser Weise der Gang der Geschichte weiter verdichtet.

Bei den Entscheidungsverfahren ist, um ein Beispiel zu nennen, der Prozess der Ausweitung des Mitentscheidungsverfahrens (jetzt ordentliches Gesetzgebungsverfahren) fortgeführt und sogar noch intensiviert worden: Es gilt nun für rund 80 Prozent der EU-Gesetzgebung, darunter auch die ursprüngliche GAP. Damit werden einige der signifikantesten Merkmale der ,traditionellen ' GM maßgeblich verändert - und die einst vertrauten Unterscheidungen verwischt, wie sie zwischen den ausschließlichen und den geteilten Zuständigkeiten der Europäischen Union bestehen.

Die Anwendung des Verfahrens qualifizierter Mehrheitsentscheidungen wurde ebenfalls ausgeweitet und stellt nun den Regelfall dar, mit einigen weiterbestehenden wichtigen Ausnahmen. Allerdings zeigen alle verfügbaren empirischen Datensätze aus Studien zum Wahlverhalten im Rat, ${ }^{10}$ dass die Beschlussfassung mit qualifizierter Mehrheit eher zur Abschreckung von Blockierern dient und nicht zur Herbeiführung von Entscheidungen. Mit anderen Worten, tendenziell bevorzugen die Mitgliedstaaten die Entscheidungsfindung im Konsens - selbst wenn diese durchaus auch anders getroffen werden könnte, wie etwa in der Handelspolitik. Und sie machen von einer Abstimmung mit qualifizierter Mehrheit (in der Regel bei verteilungspolitischen und manchmal auch bei regulatorischen Angelegenheiten) nur dann Gebrauch, wenn es vor allem innenpolitischen Zwecken dient. ${ }^{11}$

Wie bereits oben erwähnt, diente die verstärkte Zusammenarbeit ebenfalls als Abschreckungsmittel gegen Blockaden und zur Erleichterung des Entscheidungsprozesses.

Die konstruktive (qualifizierte) Enthaltung ihrerseits wurde seit ihrer Einführung mit dem Vertrag von Amsterdam nur einmal angewandt - nämlich als die ,EU-27‘ im Februar 2008 die EULEX-Mission im Kosovo ins Leben gerufen hat (und Zypern sich der Stimme enthielt). Interessanterweise gilt sie heute aber als ein Instrument im Rahmen des sogenannten Verfahrens ,im gegenseitigen Einvernehmen', wie es jüngst für den Euro-Plus-Pakt beschlossen wurde.

Es zeigt sich also, dass das Bild hinsichtlich der Methoden sogar noch weniger eindeutig ist als zu vermuten war. Wenn die Unterschiede bereits zuvor schon weniger in der Natur der Sache als in deren Abstufung begründet waren, ${ }^{12}$ dann sind sie jetzt noch verschwommener und zunehmend fraglich.

Auf der einen Seite - so banal es auch klingen mag - hat der Vertrag der Union die volle Rechtspersönlichkeit verliehen und damit indirekt die Europäische Gemeinschaft, aufgelöst'. Zumindest formal, daher ist es eigentlich unsachgemäß, weiterhin auf die GM zu verweisen. Ein bisschen weniger formell betrachtet, lässt sich festhalten, dass die GM am besten funktionierte, wenn sich die Mitgliedstaaten im Vorfeld auf die politischen Ziele, die mittels der GM verfolgt werden sollten, verständigt hatten - als Mittel für einen gemeinsa-

10 Vgl. zum Beispiel Fiona Hayes-Renshaw/Helen Wallace: The Council of Ministers, Basingstoke/New York, 2006, S. 259 ff.

11 Deutschland ist unter den Mitgliedstaaten dafür das bekannteste Beispiel. In den 1990er Jahren zählte es bei Abstimmungen im Rat zu den häufigsten ,Verlierern'. Eine Abstimmung zu initiieren und (sichtbar) in der Minderheitenposition zu sein, diente einzig und allein dazu, den nationalen Akteuren zu zeigen, dass die Regierung die spezifischen nationalen Interessen (wenn auch vergeblich) verteidigt hat, während gleichzeitig das übergeordnete gemeinsame/allgemeine Interesse - wie auch von Deutschland vorgegeben - die Oberhand gewann.

12 Siehe die ausgezeichnete Untersuchung von Philippe de Schoutheete: Mode de decision dans l'Union, Notre Europe: Les Brefs Nr. 24, März 2011. 
men Zweck anstelle eines magischen Instruments, um grundlegende Differenzen zu überwinden. Ebenso lässt sich sagen, dass eine solche Übereinkunft leichter zu erreichen war in einer Europäischen Union der sechs, neun oder zwölf Mitgliedstaaten als in der Europäischen Union der 15, 25 oder 27 Mitgliedstaaten. In anderen Worten: Die zunehmende ,Hybridisierung ' und die gegenseitige Beeinflussung und Verflechtung von Methoden ist auch dem Zusammenwirken dieser Faktoren sowie der abnehmenden Konvergenz der Politiken und der sinkenden Homogenität innerhalb der Europäischen Union geschuldet.

Auf der anderen Seite werden die gängigen Ansichten und herkömmlichen Ansätze, vor allem im Bereich der Außenpolitik und Außenbeziehungen, herausgefordert durch die mehrfache Rolle von der Hohen Vertreterin der Union für Außen- und Sicherheitspolitik Catherine Ashton sowie dem ihr zuarbeitenden dreiarmigen Europäischen Auswärtigen Dienst (EAD). ${ }^{13}$ Und selbst das Amt, das Herman Van Rompuy als Präsident des Europäischen Rates ausfüllt (und seine Art es zu interpretieren), könnte - zumindest substanziell - das Profil der ,traditionellen` IM infrage stellen, wofür es auch schon zu Solanas Zeiten Anzeichen gab.

Selbstverständlich gibt es auch noch andere plausible Gründe für die zunehmende Diversifizierung und die steigende Zahl unterschiedlicher Methoden sowie für den wachsenden Bedeutungsverlust der alten GM.

Zunächst einmal ist die Änderung der Zusammensetzung der Kommission zu nennen seit 2004 stellt jeder Mitgliedstaat einen Kommissar -, dies hat de facto zu weniger politischem Einsatz seitens der größeren Mitgliedstaaten geführt, die bis zu diesem Zeitpunkt je zwei Kommissare ins Kollegium entsandten (und dies häufig aus unterschiedlichen politischen Lagern, was noch zur Steigerung der Legitimität beitrug). Gerade die zunehmende Distanzierung Deutschlands kann ebenso auf das daraus entstandene Ungleichgewicht zurückzuführen sein, das zwischen dem formalen Einfluss im Kollegium (der jetzt dem der kleinen und kleinsten Staaten entspricht) und seinem beachtlichen Gewicht, gemessen an Bevölkerungszahl und Bruttoinlandsprodukt, besteht. Dieses wird heute sowohl im Europäischen Parlament als auch von den neuen Abstimmungsmodalitäten im Rat (ab 2014) wiedergegeben - aber eben nicht mehr in der Kommission.

Darüber hinaus wurde die Entscheidungsfindung in der Europäischen Union vor allem in den letzten Jahren insgesamt immer, präsidentieller'. Dies zeigt die Institutionalisierung des Europäischen Rates als eigenständiges Organ durch den Vertrag von Lissabon, genauso wie die gegenwärtige Verlagerung der politischen Entscheidungsfindung von den wichtigen Ratsformationen (insbesondere der Rat für Allgemeine Angelegenheiten und Auswärtige Angelegenheiten (GAERC) und nun ebenfalls der Rat für Wirtschaft und Finanzen, ECOFIN) hin zu den Gipfeltreffen der Staats- und Regierungschefs - die tatsächlich immer häufiger geworden sind. Eine derartige zunehmende Politisierung und die öffentliche Zurschaustellung haben die GM weiter geschwächt, die geschaffen wurde, um scheinbar technische Lösungen für politische Probleme zu entwerfen und politische Entscheidungen eher langals kurzfristig anzulegen.

Letztendlich wird es der wachsende Machtzugewinn des Europäischen Parlaments sein, der wahrscheinlich alle Methoden weit stärker beeinflussen wird, als bisher angenommen. Nicht nur haben die Mitglieder des Europäischen Parlaments gerade erst begonnen, von ihren neuen Befugnissen offensiver Gebrauch zu machen, auch wurden einige von diesen eingesetzt, um die Möglichkeit des Erwerbs weiterer Befugnisse zu erkunden. Dies hätte - im

13 Für eine vorläufige Bewertung siehe Antonio Missiroli: The EU 'Foreign Service': Under Construction, Robert Schuman Centre for Advanced Studies, European University Institute: RSCAS Policy Papers 4/2010. 
,methodischen' sowie systemischen Sinn - weitreichende Folgen für alle anderen Akteure im EU-Institutionengfüge, und würde zu unerwarteten Ergebnissen und unbeabsichtigten Folgen führen.

\section{Ist die Geschichte von den zwei Methoden zu Ende?}

Insofern oberflächlich und vor allem als unangemessen erscheint der aktuelle öffentliche Diskurs über die Methoden und die daraus resultierende Polarisierung zwischen GM und IM - mit Merkels UM als unbekannter Dritten. Die Debatten wirken künstlich und vor allem als taktische Manöver (unabhängig von den guten Absichten und der Aufrichtigkeit einiger Diskutanten). Darüber hinaus bergen sie auch die Gefahr, die eigentlichen Themen der politischen Debatte zu verschleiern, und sie zwingen die beteiligten Akteure Partei zu ergreifen. Dies führt letztlich dazu, dass die Herbeiführung eines tragfähigen Konsenses und die Durchsetzung einer wirksamen Politik in noch weitere Ferne rücken.

Zwei Punkte sind besonders hervorzuheben. Erstens: Die Spannungen zwischen GM und IM waren zu keinem Zeitpunkt ein Nullsummen-Spiel und sollten es auch jetzt nicht werden. Unbestritten, die Juristischen Dienste des Rates und der Kommission scheinen manchmal zu Präventivschlägen anzusetzen und Grabenkämpfe auszutragen - und (so) als ,Hüter der Methoden' aufzutreten, wenn es sich um Kompetenzfragen handelt. Doch die politischen Instrumente sind nicht nur einer Seite zuzuordnen und aufgrund des bloßen Ausmaßes der aktuellen Herausforderungen sind sie alle unverzichtbar. Die Aufmerksamkeit sollte daher dem am besten geeigneten ,Mix‘ gelten, und nicht der relativen Größe und Sichtbarkeit der einzelnen Bestandteile.

Zweitens: Selbst wenn man die GM an einem Ende des Spektrums und die IM auf der gegenüberliegenden Seite - in einer Art idealtypischen Kontinuums - anordnen würde, zeichnet sich in der Realität ein anderes Bild ab: In der Praxis der EU-Politik zeigt sich nämlich, dass sich die tatsächlich angewandten Verfahren häufig zwischen und, was noch wichtiger ist, weiterhin entlang dieser Linie bewegen und fortentwickeln. Es ist wahrlich kein Geheimnis, dass die europäische Integration im Laufe der Zeit verschiedene Wege geprüft und verworfen beziehungsweise übernommen hat - und das mit und nach dem Vertrag von Lissabon die alte Garnitur von (mehr oder weniger) unterschiedlichen Methoden endgültig keinen Bestand mehr hat.

Folglich sollte der recht vage Hinweis von Bundeskanzlerin Merkel auf eine sich im Entstehen befindende UM - leider wieder einmal im Singular - eher als eine Einladung begriffen werden, um die Folgen der fortwährenden Ausdehnung der EU-Bereiche mit geteilter Zuständigkeit auf den Entscheidungsprozess der Europäischen Union zu überdenken. Ihr Hinweis könnte auch als Gelegenheit verstanden werden, die sich aktuell vollziehende Ausweitung der ,hybriden“ Verfahren zu vereinheitlichen und die Fortdauer der alten Pfeilerstruktur - trotz ihrer formalen Bändigung durch den Vertrag von Lissabon - und deren Belastbarkeit neu zu bewerten.

Die Anhänger der GM betrachten die UM als Wolf im Schafspelz, doch eigentlich ist die UM kaum mehr als ein leeres Blatt, vor allem nach den unvermeidlichen Zugeständnissen an das deutsche Bundesverfassungsgericht 2009. Die eigentliche Debatte (und Kontroverse) sollte daher darauf ausgerichtet werden, wie sich dieses leere Blatt mit Erfolgsrezepten füllen lässt, die effektiv und vorteilhaft für den gesamten Integrationsprozess sind.

Deshalb ist eine starke Dosis Pragmatismus und Offenheit absolut unerlässlich - wenn auch wahrscheinlich nicht ausreichend. 
Der ehemalige US-Präsident Bill Clinton wies kürzlich darauf hin, dass das 21. Jahrhundert einen ,whatever works"-Ansatz erfordert. In und für die Europäische Union muss jedoch solch ein nackter Pragmatismus mit handfesten Beweisen für die Wirksamkeit einer solchen Politik kombiniert werden - was je nach Bereich(-en) leicht unterschiedliche Handlungsrezepte erfordern kann -, und sollte tragfähige vertrauensbildende Maßnahmen zwischen den Institutionen, Mitgliedstaaten und auch Bürgern umfassen. Gegenseitiges Vertrauen und gemeinsame Entschlossenheit sind wesentliche Bestandteile für jedes aktuelle oder künftige Erfolgsrezept und insbesondere ersteres - das gegenseitige Vertrauen - wurde durch die Staatsschuldenkrise stark in Mitleidenschaft gezogen.

Eines aber ist sicher: Eine anhaltende Kontroverse über die eine optimale Methode - als ob es einen ,one size fits all'-Ansatz für alle komplexen Herausforderungen unserer Zeit geben würde - ist nutzlos und sogar kontraproduktiv. Es ist allerdings bemerkenswert, dass in der jüngsten Debatte über die Reform der wirtschaftspolitischen Steuerung in der Eurozone, die einen (Niederlande, Finnland, Belgien) für eine stärkere Regulierung und Gemeinschaftskompetenz, die anderen (Frankreich, manchmal auch Deutschland) für eine überwiegend intergouvernementale Struktur plädieren. Funktionelle wie auch politische Überlegungen spielen hier eine eindeutige Rolle; das Endergebnis bleibt aber noch offen. ${ }^{14}$

Kein klassischer oder zeitgenössischer Philosoph scheint in der Lage zu sein, für diesen besonderen Bedarf eine überzeugende Methode anbieten zu können, möglicherweise mit Ausnahme von Karl Popper und seiner evolutionären Erkenntnistheorie - wonach nicht nur unser Wissen, sondern auch unsere Ziele und Ansprüche durch die kritische Überprüfung in einem fortwährenden Prozess von „Versuch und Irrtum“ wachsen. ${ }^{15}$

Will man weitere Überlegungen anstellen, dürfte es daher an dieser Stelle klüger sein, bei Leo Tolstois „Anna Karenina“ (1877) und dessen berühmten ersten Satz Zuflucht zu suchen: „Alle glücklichen Familien sind einander ähnlich; aber jede unglückliche Familie ist auf ihre besondere Art unglücklich.“

Das Familienleben in der Europäischen Union liegt irgendwo in der Mitte: Während die Vorstellung einer Methode für alle Fälle zumindest heute als ein unerreichbares Ziel erscheint, bleibt das Streben nach einem (gemeinsamen) Glück ein legitimes und lobenswertes Ziel.

14 Kommissionspräsident José Manuel Barrosos jüngster Ruf nach einer Stärkung der GM (in seiner Rede zur ,State of the Union' am 28. September 2011 im Europäischen Parlament) gilt als (völlig verständlicher) Appell für mehr politische Kohäsion und mutigere Übertragung von administrativer und regulativer Kompetenz zu gemeinschaftlichen Institutionen - er bleibt aber noch vage über die präzisen Konturen dieser Übertragung im spezifischen Kontext der Wirtschafts- und Währungsunion.

15 Dargelegt in seinen Vermutungen und Widerlegungen (1963), die allgemein als Fundament des „kritischen Rationalismus" und des liberalen erkenntnistheoretischen Ansatzes gelten. 